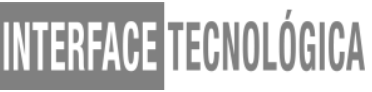

\section{O PAPEL DO APQP (ADVANCE PRODUCT QUALITY PLANNING) NO PRODUTO FINAL COM FOCO NO CLIENTE}

\section{THE ROLE OF APQP (ADVANCE PRODUCT QUALITY PLANNING) IN THE FINAL PRODUCT WITH A FOCUS ON THE COSTUMER}

\author{
Oriel Alan Schineider - oriel.schineider@hotmail.com \\ Faculdade de Tecnologia (Fatec) - Taquaritinga - SP - Brasil \\ Angelita Moutin Segovia Gasparotto - angelita.gasparotto@fatecq.edu.br \\ Faculdade de Tecnologia (Fatec) - Taquaritinga - SP - Brasil
}

DOI: $10.31510 /$ infa.v17i2.998

Data de publicação: 18/12/2020

\section{RESUMO}

O presente artigo retrata sobre o método APQP (Advance Product Quality Planning) com o objetivo de explorar em como é aplicado por empresas do ramo automotivo, descrevendo sua definição, como pode ser aproveitado e suas fases para aplicação. É um método que guia e possibilita a verificação das etapas no processo de desenvolvimento ou alteração de um produto. Esse processo envolve vários departamentos da empresa, possibilitando que o produto satisfaça o cliente, visando qualidade e redução de custos desde a fase de planejamento até o lançamento do item, avaliando possíveis falhas no processo. A presente pesquisa é considerada bibliográfica e descritiva e para a exemplificação do uso do APQP foi descrito um estudo retirado de um artigo científico que demonstra na prática como uma organização automobilística aplica esse recurso. Sobre essa perspectiva, o APQP é apontado como uma ferramenta muito útil que contribui para as empresas alcançarem seus objetivos organizacionais e também auxilia para a melhoria contínua em seus processos de qualidade.

Palavras-chave: APQP. Qualidade. Etapas de Desenvolvimento do Produto. Satisfação do cliente.

\begin{abstract}
This article reflects on the method APQP (Advanced Product Quality Planning) in order to explore how it is applied by automotive companies, describing its definition, how it can be used and its stages for application. It is a method that guides and makes it possible to verify the steps in the process of developing or changing a product. This process involves several departments of the company, allowing the product to satisfy the customer, aiming at quality and cost reduction from the planning phase to the launch of the item, evaluating possible failures in the process. The present research is considered bibliographic and descriptive and to exemplify the use of the APQP, two case studies from a scientific article have been described that demonstrate in practice how automobile organizations apply this resource. From this
\end{abstract}


perspective, APQP is seen as a very useful tool that helps companies achieve their organizational goals and also assists for continuous improvement in their quality processes.

Keywords: APQP. Quality. Product Development Steps. Customer Satisfaction.

\section{INTRODUÇÃO}

Nos últimos tempos as empresas têm vivido grandes momentos com a atualização de tecnologia e grande sortimento de projetos, o que faz com que cada empresa busque produzir um produto que tenha um diferencial em destaque para oferecer. Esse diferencial deve estar unido com a qualidade do produto. Para que as organizações sejam capazes de competir no mercado atual elas devem possuir a capacidade de produzir com qualidade. O termo Qualidade está relacionado a vários fatores, dentre eles características físicas dos produtos, conformidade ao custo, qualidade relacionada a produzir e entregar com as características e necessidades do cliente e serviços de entrega e garantia (LOBO, 2020).

De acordo com Oliveira (2003) as empresas vivem atualmente a era da Qualidade Total, na qual o foco são as expectativas e imposições dos clientes. Outro ponto é que todos os setores da empresa são responsáveis por produzir um produto ou serviço de qualidade e para isso é indispensável que todos os processos estejam concernentes a Gestão da Qualidade e sejam considerados em todos os níveis da organização.

Devido ao momento em que as empresas estão inseridas, é necessário o auxílio de ferramentas adequadas para a execução de projetos muito diversificados. Em vista disso, muitas ferramentas e métodos da Qualidade são usados nos dias de hoje para facilitar e aperfeiçoar os processos de produção e tomada de decisão. Um recurso muito significativo usado por indústrias automotivas é o APQP (Advanced Planning for Product Quality) que em português significa "Planejamento avançado da qualidade do produto". Essa ferramenta tem por função estipular os procedimentos e prazos nas fases da fabricação do produto, junto com o responsável. Esse recurso é de suma importância, pois o processo de desenvolvimento do produto, também conhecido como PDP, exige uma gestão estruturada, porquanto ao final de seu desenvolvimento é fundamental que o produto esteja aprovado e que as características de produção sejam conservadas preservando a qualidade do produto final e que auxiliará para a produção de outros itens (ROCHA; SALERNO, 2014). 


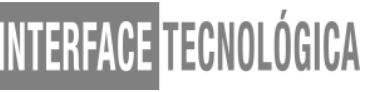

Diante disso, é possível compreender a importância dessa ferramenta para a gestão de produção e o quanto as empresas que a colocam em prática aperfeiçoam seus projetos, pois o APQP sendo uma ferramenta da indústria automotiva que tem o foco voltado para os inúmeros estágios de elaboração e desenvolvimento de um produto se torna primordial para realizar a satisfação do cliente, pois a qualidade de um produto já começa no seu desenvolvimento.

Portanto, o presente trabalho tem por objetivo explorar sobre a ferramenta para que possa ser aplicada nos produtos finais durante o processo produtivo, procurando sempre atingir as expectativas do cliente com um produto de qualidade.

\section{FUNDAMENTAÇÃO TEÓRICA}

\subsection{Processo de Desenvolvimento do Produto}

O PDP (Processo de Desenvolvimento do Produto) é um conjunto de atividades utilizado em indústrias automotivas que tem o objetivo de criar um processo para o desenvolvimento do item a ser elaborado. Um fator importante que é considerado no PDP é a qualidade do produto a ser produzido, juntamente com o seu processo, pois é através dela que se pode mensurar o desempenho do processo, tempo de desenvolvimento, qual será o custo para produzi-lo sendo relevante ressaltar que as organizações buscam por um custo menos elevado (FERREIRA; TOLEDO, 2016).

Além disso, produzir um item com um padrão de qualidade e garantir que perdure nos próximos processos de novos produtos permitem as empresas automotivas que adotam essa medida estarem à frente no mercado e por isso é importante essas empresas estabelecerem relação com seus clientes, pois desta forma ela produzirá atendendo os seus requisitos específicos, evitando erros de montagem e retrabalho e diminuindo a margem de erros. Também com o processo de desenvolvimento do produto bem definido a organização permite um diferencial estratégico, impactando no custo e lucro final da empresa perante o produto.

O PDP é dividido em três etapas. A primeira se refere ao Pré-desenvolvimento. Nela os responsáveis buscam entender e atender o que o mercado almeja e será definido o produto a ser desenvolvido junto com a avaliação do custo do projeto, riscos e indicadores de monitoramento. A segunda etapa é chamada de Desenvolvimento, e nela serão exploradas as 


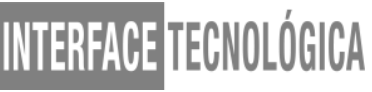

informações do projeto junto com o cliente, aplicabilidade e funções do produto, definição de quais materiais serão utilizados para produzir o projeto, suas dimensões, formas, processo de montagem, entre outros; e ao final dessas fases é feito o lançamento do produto. Por fim, a terceira etapa é chamada de Pós-desenvolvimento e nela é feito o delineamento de como o produto será acompanhado. Ademais, segundo os autores Faria at. all (2008, p.06) nesta etapa “definem-se as equipes e os recursos necessários para as alterações de engenharia, visando correções de potenciais falhas e/ou adição de melhorias requisitadas pelo cliente".

Estas etapas têm por objetivo delinear todo o processo de fabricação de um item para assim fornecer um produto que tenha menor custo, que esteja de acordo com as especificações do mercado e no tempo estipulado. Com o PDP é possível garantir padronização dos itens, planejamento de produção e diminuição de falhas.

\subsection{Contextualização do APQP}

O APQP é conhecido como um método da indústria automotiva que tem o seu objetivo voltado para os diversos estágios de desenvolvimento e aperfeiçoamento de um produto. Segundo Pimenta (2009, p.15) o APQP pode ser estipulado como "um método estruturado para definir as ações necessárias para um PDP, a fim de assegurar que um produto satisfaça o cliente." Utilizando essa ferramenta espera-se que ao final do processo de fabricação do produto todas as atividades que foram estabelecidas tenham sido realizadas de forma que o produto fabricado tenha qualidade e que a sequência de operações permaneça no modo em que foi estruturada permitindo dessa maneira que as peças que forem fabricadas posteriormente possuam as mesmas características de qualidade das anteriores.

O APQP utiliza ferramentas preventivas da qualidade e através dessa metodologia é possível oferecer um produto de qualidade, no prazo estipulado e também com o custo esperado, pois todas as operações de adaptação e alteração do projeto são realizadas antecipadamente evitando assim, gastos e retrabalhos desnecessários (ALBERTIN; GUERTZENSTEIN, 2018). Além disso, o APQP tem como propósito servir como um guia no processo de desenvolvimento e também estabelecer o melhor jeito de compartilhar resultados, informações e consequências entre fornecedores e empresas automotivas.

As indústrias automobilísticas buscam desenvolver seus projetos de peças com qualidade, visando o menor custo e dentro do prazo acordado. Diante disso, alguns dos 


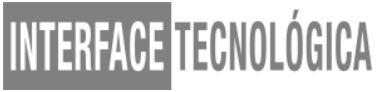

benefícios de se utilizar o APQP são "direcionar os recursos para satisfazer o cliente, promover a identificação antecipada das alterações necessárias, evitar alterações de última hora e fornecer um produto de qualidade dentro do prazo a um custo reduzido" (IRION; SOUZA, 2013, p.180). Entretanto, o APQP não deve ser considerado como um cronograma, mas sim um orientador que auxilia na validação do produto e do processo e que apresenta prazos de conclusão de atividades que são consideradas suportes ao projeto como qualidade do produto e processo (ROCHA; SALERNO, 2014).

O APQP foi desenvolvido por especialistas de grandes empresas como Ford, General Motors (GM) e Chrysler que observaram durante alguns anos os métodos de produção empregados no Japão, Estados Unidos e Europa, cujo principal objetivo era ser um método para a criação de um item e um facilitador de comunicação entre os envolvidos na elaboração do projeto. Essa ferramenta esteve contida na norma ISO/TS: 16:949:2002 cujo propósito era o desenvolvimento de um Sistema de Gestão da Qualidade que fosse igual para todos frisando a melhoria contínua e o processo de produção com o menor número de defeitos (PIMENTA, 2009).

Dentro do APQP estão inclusos os processos de: Método de Trabalho, Análise de Viabilidade, Processo de Comercialização, de Engenharia, de Qualidade e o Processo de Logística (IRION; SOUZA, 2013). Ainda, os autores complementam que é necessária a realização de documentos como o Diagrama de Fluxo que contem como é feita a operação dos processos; FMEA de Processo e Produto; Plano de controle; Instrução para o operador; Aprovação de embalagem; Produção do Lote Piloto; Validação do produto; PPAP e por fim o Início de Produção.

Portanto, percebe-se que o APQP é uma metodologia detalhada, que contribui e estabelece etapas para o desenvolvimento do produto que auxiliarão as empresas a alcançarem seus objetivos de qualidade, custo e prazo. Essa ferramenta é aplicável em toda a base de fornecimento e em todos os relacionamentos entre cliente e fornecedor e determina as entradas e saídas necessárias de cada estágio do processo de desenvolvimento do produto.

\subsection{Etapas do APQP}

O APQP é dividido em etapas que auxiliam para a utilização desse método. E para que o APQP seja eficiente, segundo o autor Ferraresi (2015, p. 52) "todo o sucesso do 
desenvolvimento depende das informações coletadas neste início e de como serão analisadas, chegando a uma definição final sobre o problema, o que se pretende atingir e/ou resolver com o produto, seguindo durante o ciclo de vida do mesmo". Logo, entende-se que é preciso seguir corretamente todas as fases do APQP além de ser imprescindível ter todos os planos e objetivos traçados desde o planejamento na sua primeira fase.

A primeira etapa é o Planejamento, que consiste em identificar e analisar as necessidades e expectativas do cliente. É nessa etapa que se estabelece as atividades necessárias, o responsável, o cronograma para o processo, a escolha da equipe, o conceito do produto, áreas envolvidas, taxa de não conformidade e exigências dos clientes (ALBERTIN; GUERTZENSTEIN, 2018). Além disso, são consideradas nessa fase a suposição da confiabilidade, objetivo e qualidade do produto, lista de materiais, possíveis eventualidades relacionadas ao produto e objetivos do projeto (FERRARESI, 2015). Também é possível considerar a voz do consumidor através de reclamações, recomendações e conceitos obtidos dos clientes através de pesquisa de mercado, experiência da equipe e informações de qualidade.

Na segunda fase, que é Projeto e Desenvolvimento do Produto, é feita a análise do efeito e modos de falha do produto, o plano de controle, onde é descrito o sistema de fabricação com os atributos do produto e do processo, técnica de medição, plano de amostragem, plano de reação e especificações de materiais (ALBERTIN, 2018). Além disso, os elementos desta fase consistem em uma reavaliação crítica dos requisitos, prazos, objetivos e metas do processo, a partir de uma análise que considere todos os fatores do projeto, mesmo que sejam de propriedade do cliente, a fim de concluir a avaliação sobre a viabilidade da produção (PIMENTA, 2009).

Projeto e Desenvolvimento de Processo é a terceira etapa, e nela os requisitos e levantamento de potenciais problemas são revisados, juntamente com a etapa de comprometimento da equipe com a ajuda e suporte da gerência. Além disso, é estipulado como será feito a administração do produto ao longo de seu processo de fabricação e também garantir que as exigências do cliente sejam atendidas (PIMENTA, 2009).

Na visão de Albertin (2018, p.55):

Com as informações da etapa do PDP, são realizados: Plano do sistema de medição; definição dos padrões e especificações da embalagem; revisão do diagrama de fluxo do produto e plano de controle; layout das instalações; FMEA do produto; instruções do processo; plano de manutenção; estudos de capacidade do processo; análise 


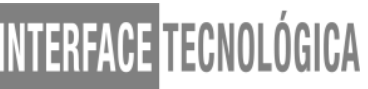

crítica da gerência; dispositivos à prova de falhas; sistemas de controle de linha e outros.

$\mathrm{Na}$ quarta etapa Validação do Produto e do Processo, o método de validação é alcançado através da fabricação de um lote piloto, no qual as peças serão medidas, a capacidade de produção será verificada junto com a diversificação das propriedades principais do produto e a qualidade do sistema de medição (ALBERTIN, 2018). Também, de acordo com o autor Ferraresi (2015) são verificadas as instruções do processo, layout de planta, avaliação da embalagem e plano de controle pré-lançamento. Conjuntamente é validado se o produto desenvolvido atende as necessidades do cliente e se os resultados estão condizentes com as expectativas do mesmo.

$\mathrm{Na}$ última fase, chamada de Retroalimentação, Avaliação e Ação Corretiva, estão relacionadas a melhoria contínua e satisfação do cliente. Também serão apontadas e registradas as lições aprendidas que auxiliarão para a fabricação dos próximos produtos. É efetuada uma avaliação para verificar se todos os estágios atendem as exigências e determinações dos clientes e se necessário é realizada ações corretivas e após isso, inicia-se o fornecimento de peças para os consumidores (PIMENTA, 2009).

\section{PROCEDIMENTOS METODOLÓGICOS}

O presente artigo utilizou a pesquisa bibliográfica como metodologia de pesquisa, que segundo Gil (2008, p. 50) “é desenvolvida a partir de material já elaborado, constituído principalmente de livros e artigos científicos". A autora Bortoloti $(2015$, p. 73$)$ também cita que esse tipo de pesquisa "em relação aos procedimentos de coleta, caracteriza-se pela metodologia que elege a fonte bibliográfica como recurso para explicar um problema, conhecer e analisar as contribuições sobre determinado assunto" e possui também características da pesquisa descritiva na qual seu principal objetivo é a "descrição das características de determinada população ou fenômeno ou estabelecimento de relações entre variáveis" (GIL, 2008, p. 28).

Da mesma maneira, o trabalho apresenta um estudo de caso coletado através de um artigo científico com a finalidade de demonstrar como esse método é aplicado. O estudo de caso é definido como um tipo de pesquisa que busca analisar e descrever um determinado caso. Seu objetivo "visa a investigação de um caso específico, bem delimitado, 
contextualizado em tempo e lugar para que se possa realizar uma busca circunstanciada de informações" (VENTURA, 2007, p. 384).

\section{RESULTADOS E DISCUSSÃO}

\subsection{Estudo sobre a alteração de um produto em uma montadora}

O estudo demonstrado foi extraído do artigo científico publicado pelos autores Rocha e Salerno (2014, p. 236 - 239). A empresa participante dessa pesquisa está localizada no Brasil e o projeto do produto foi desenvolvido entre 2008 e 2009. Esse projeto aborda sobre a alteração de um produto já existente para atender a um novo requisito estipulado.

$\mathrm{O}$ presente estudo retrata "o desenvolvimento de produto inserido em projeto de pequeno porte dentro da montadora, que implica na inclusão de uma nova função para o usuário final" (ROCHA; SALERNO, 2014, p. 236). Para isso, o fornecedor que é chamado de A1 precisa alterar o sistema elétrico que já era fornecido para a montadora com o intuito de mudar outros conjuntos interligados para que fosse integrada a nova função no veículo. $\mathrm{O}$ prazo estipulado para entrega foi de sete meses, iniciando a partir da oficialização contratual até o encerramento do processo de validação do produto e entrega de PPAP e PSW que é a certificação de submissão de peça.

Durante o estudo, foram identificados vários erros que haviam sido cometidos e que não atendiam as fases do APQP. Na ilustração abaixo, é possível identificar as falhas e o que elas correspondiam no método do APQP.

Ilustração 1 - Falhas identificadas no Estudo de Caso

\begin{tabular}{|c|c|}
\hline Falhas identificadas & Fases do APQP \\
\hline Atraso na admissão oficial do Fornecedor no projeto & Planejamento \\
\hline $\begin{array}{l}\text { Demora nas definições de Engenharia e início de construção do } \\
\text { ferramental. }\end{array}$ & $\begin{array}{l}\text { Desenvolvimento do produto e } \\
\text { processo }\end{array}$ \\
\hline Falha nas ações de conclusão de um projeto. & $\begin{array}{l}\text { Validação do produto e/ou processo/ } \\
\text { Retroalimentação. }\end{array}$ \\
\hline $\begin{array}{l}\text { Falha no planejamento do prazo para desenvolvimento de uma } \\
\text { nova tecnologia nos fornecedores. }\end{array}$ & Planejamento \\
\hline Interferência de fatores externos às estratégias da montadora. & Planejamento \\
\hline Protótipos não refletem o produto final. & Projeto e desenvolvimento do produto. \\
\hline Primeiras peças de produção não foram validadas. & Validação do Produto e do Processo. \\
\hline $\begin{array}{l}\text { APQP substituindo referências de prazos do cronograma da } \\
\text { montadora. }\end{array}$ & Planejamento \\
\hline $\begin{array}{l}\text { Redução forçada dos prazos de validação do produto e processo } \\
\text { dentro do fornecedor. }\end{array}$ & $\begin{array}{l}\text { Planejamento/ Validação do Produto e do } \\
\text { Processo. }\end{array}$ \\
\hline
\end{tabular}


Fornecedor trabalha antecipadamente, sem contrato formal com a montadora, assumindo riscos para atender os prazos.
Erro cometido antes do processo do APQP

Fonte: Adaptado de Rocha, Salerno (2014, p. 239)

O primeiro erro decorreu logo no início do processo, pois houve um atraso de quarenta dias para oficialização de entrada do fornecedor no projeto. Além disso, o segundo erro deu-se por atrasos na fase inicial do projeto que ocorreram na montadora e por fim comprometeram outras etapas, como o tardamento de definições de engenharia que afetaram os acertos comerciais e definições como ferramental, produção, matéria-prima e dispositivos.

O motivo da terceira falha foi causado por outros processos pendentes de produtos anteriores e que não estavam adequadamente finalizados. De acordo com os autores Rocha e Salerno $(2014$, p. 237) "a causa desse problema pode estar na falha de simulações com protótipos, onde poderiam ter sido identificados problemas de montagem e ergonomia ou também falha no processo de retroalimentação e finalização do projeto".

No quarto erro foi identificado que novamente houve atrasos por parte da engenharia em definir outros componentes que envolviam nova tecnologia. Posteriormente a quinta falha sucedeu-se porque esse novo projeto foi desenvolvido perante a regulamentação governamental e havia a expectativa dessa proposta ser procrastinada.

Conjuntamente, as atividades relacionadas ao protótipo não estavam realizadas no tempo estipulado, causando o sexto problema. Como muitas atividades não estavam efetuadas de acordo com o cronograma da montadora, os fornecedores entregaram peças não validadas e com o consentimento da engenharia que ocasionou a sétima dificuldade.

O oitavo problema aconteceu devido a montadora ter perdido os períodos de entrega definidos em seu cronograma e por isso ela passou a seguir os prazos determinados pelo fornecedor. As duas últimas complicações decorreram porque os prazos ao longo do processo todo não foram obedecidos e por isso houve redução do tempo de entrega do PSW e o fornecedor só conseguiu entregar o projeto dentro dos sete meses estabelecidos porque ele começou a trabalhar antes da formalização contratual.

Conclui-se, portanto, que muitas dificuldades foram encontradas durante todo o processo do APQP, iniciando pela fase de Planejamento até a fase de Lançamento. Todavia, foi possível perceber como a definição dos prazos, a correta documentação e o acordo entre a montadora e o fornecedor foi importante para que fosse possível cumprir o prazo de entrega final, minimizando custos imprevistos e garantindo a qualidade. Esse método, como visto 


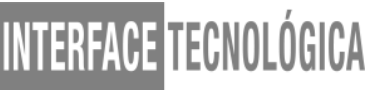

anteriormente pelo autor Pimenta (2009) contribui para o processo de desenvolvimento do produto, auxiliando quanto aos prazos de conclusão e para a satisfação do cliente. Ele auxilia também para o alcance de qualidade e menor custo para a empresa. Porém, para isso ser alcançado, esse método precisa ser seguido corretamente conforme acordado entre as partes e com suas fases estabelecidas.

\section{CONCLUSÃO}

Em virtude das informações apresentadas, com o objetivo de explorar sobre a ferramenta $\mathrm{APQP}$ para que possa ser aplicada durante o processo produtivo, procurando sempre atingir as expectativas do cliente com um produto de qualidade, conclui-se que esse método é de grande importância para que o produto final esteja de acordo com as especificações do cliente e proporcione sua satisfação, produzido dentro do prazo determinado, minimizando os custos e que possua todas as características de qualidade, estabelecendo os responsáveis por cada fase do APQP para que ao final de todo o processo o produto seja entregue conforme o esperado.

Esse procedimento facilita a comunicação entre os responsáveis envolvidos e estipula todas as fases que o processo de desenvolvimento do produto (PDP) deve seguir, assim como ele permite identificar alterações necessárias antecipadamente e colabora para que os futuros processos estejam validados e padronizados para a próxima produção.

Nota-se que no estudo demonstrado, houve falhas quanto a aplicação de algumas fases do APQP, como no planejamento, desenvolvimento e validação do produto e principalmente em relação ao cumprimento dos prazos desde o início do processo e percebe-se também que o fornecedor considerou o tempo imposto pela montadora como curto. Por isso, cabe aos envolvidos estabelecerem conjuntamente os prazos e todas as etapas que o processo deve percorrer para assim evitar atrasos e conflitos entre as empresas.

Para pesquisas futuras, sugere-se abranger a pesquisa de campo buscando-se analisar com mais detalhes a aplicação das fases do APQP dentro das indústrias e observar quais pontos poderiam ser melhorados ou trabalhados de outra forma. Além disso, seria proveitoso analisar o PDP de empresas que não aplicam o APQP para que seja possível ver a diferença dos processos e o resultado final, com o intuito de demonstrar a aplicabilidade, vantagens e dificuldades na utilização do Planejamento Avançado da Qualidade do Produto. 


\section{WTEERFAET TECNOLLGGCA}

\section{REFERÊNCIAS}

ALBERTIN, Marcos. Planejamento avançado da qualidade: sistemas de Gestão, técnicas e ferramentas. Rio de Janeiro: Editora Alta Books, 2018. Disponível em: https://books.google.com.br/books?hl=pt-

BR\&lr=lang_pt\&id=hxhgDwAAQBAJ\&oi=fnd\&pg=PA1\&dq=livro+apqp\&ots $=$ aHUHrjzStZ \&sig=CQFr4GJ131vz6VppB16x3-YiSDU\#v=onepage\&q\&f=false. Acesso em: 22 Jun. 2020.

APQP. Planejamento Avançado da Qualidade do Produto. Etapas do APQP, 2019.

Disponível em: https://apqp.com.br/. Acesso em: 24 Jun 2020.

BORTOLOTI, Karen Fernanda. Metodologia da Pesquisa. Rio de Janeiro: SESES, 2015. Disponível em: https://d1wqtxts1xzle7.cloudfront.net/43353708/LIVRO_PROPRIETARIOMETODOLOGIA_DA_PESQUISA.pdf?1457102146=\&response-contentdisposition $=$ inline $\% 3 \mathrm{~B}+$ filename $\% 3$ DLIVRO_PROPRIETARIO-

METODOLOGIA_DA_PESQUI.pdf\&Expires $=1593133639 \&$ Signature $=$ W2v98QnbYeFSH7 Tq Mbf9tvXdHNyvPaEbywbp31 amliDLR8ogRaF6GYCQp4E0f1 by7TznNRfb1rh3q7qgDzr zvnsYo4yQPZmZWC4Cr5DIrlLEsgu33FYic51PaK4ezjMrTCPFGndEAOOFOVAIkA9xPM X8e8z07FFE6Nm12cKN4zu6DFt9Eb9IGpXUPHgQRkAPyRzcc4WiftK43y8ap6EhZFAac2oFc6bDg9UDZSM9024Dekf5RouZxOCcrBRb5JeAP1fchuwHoYaDB1LU6RVq4Bx3jPSiVEtLWqSubaNtKbT8vEfY LktCT9snh5MPw8oWxQePnyJISJNohr6Kkw_\&Key-PairId=APKAJLOHF5GGSLRBV4ZA. Acesso em: 25 Jun 2020.

FARIA, Adriana Ferreira de et al. Processo de Desenvolvimento de Novos Produtos: Uma Experiência Didática. XXVIII Encontro Nacional de Engenharia de Produção. Rio de Janeiro, Out. 2008. Disponível em:

http://www.abepro.org.br/biblioteca/enegep2008_tn_stp_073_521_12155.pdf. Acesso em: 23 Jun. 2020.

FERRARESI, Fernando. Incorporação do Projeto de Embalagem na Ferramenta APQP. 2015. 117 f. Dissertação - Universidade Federal de Goiás, 2015. Disponível em: https://repositorio.bc.ufg.br/tede/handle/tede/5476. Acesso em 16 Jul 2020.

FERREIRA, Heloisa Souza Ribeiro; TOLEDO, José Carlos de. Metodologias e Ferramentas de Suporte à Gestão do Processo de Desenvolvimento de Produto (PDP) na Indústria de Autopeças. Universidade Federal de São Carlos - UFSCAR. In: Congresso Brasileiro de Gestão de Desenvolvimento de Produto. Vol. 3, 2001. Disponível em: https://www.researchgate.net/profile/Jose_Toledo4/publication/266907815_METODOLOGI AS_E_FERRAMENTAS_DE_SUPORTE_A_GESTAO_DO_PROCESSO_DE_DESENVOL VIMENETO_DE_PRODUTO_PDP_NA_INDŪSTRIA_DE_AUTOPECAS/links/56c6050d08 ae0d3b1b5fc107/METODOLOGIAS-E-FERRAMENTAS-DE-SUPORTE-A-GESTAO-DOPROCESSO-DE-DESENVOLVIMENTO-DE-PRODUTO-PDP-NA-INDUSTRIA-DEAUTOPECAS.pdf. Acesso em 24 Jun 2020.

GIL, Antonio Carlos. Métodos e Técnicas de Pesquisa Social. 6 ed. São Paulo: Atlas. 2008. Disponível em: https://ayanrafael.files.wordpress.com/2011/08/gil-a-c-mc3a9todos-etc3a9cnicas-de-pesquisa-social.pdf. Acesso em: 25 Jun 2020. 
IRION, Crishna; SOUZA, Flávio Carneiro. Introdução de um processo de manufatura do Planejamento Avançado da Qualidade do Produto - APQP na Indústria Automobilística. In: SILVA, Jane Aparecida de Oliveira; HANRIOT, Patrick de Morais (Organizadores).

Qualidade e produtividade dos processos organizacionais: cenários e análises. São Paulo: Editora Vale dos Livros, 2013. P. 179 - 191. Disponível em:

http://www.univas.edu.br/docs/biblioteca/Livro_ICongressoCientificodaProducao_2012.pdf\# page $=179$. Acesso em: 22 Jun. 2020.

LOBO, Renato Nogueirol. Gestão da Qualidade. 2 ed. São Paulo: Érica, 2020. Disponível em https://books.google.com.br/books?hl=pt-

$\mathrm{BR} \& 1 \mathrm{r}=\& \mathrm{id}=\mathrm{S} 8 \mathrm{y} 8 \mathrm{DwAAQBAJ} \& \mathrm{oi}=\mathrm{fnd} \& \mathrm{pg}=\mathrm{PT} 18 \& \mathrm{dq}=\mathrm{gest} \% \mathrm{C} 3 \% \mathrm{~A} 3 \mathrm{o}+\mathrm{da}+$ qualidade $\& \mathrm{ots}=$ BRnVtcZ9uh\&sig=xJq7ckVUI3cJRJ6CVm41GM-

$\mathrm{hF} 5 \mathrm{Y} \# \mathrm{v}=$ onepage $\& \mathrm{q}=$ gest $\% \mathrm{C} 3 \% \mathrm{~A} 3 \mathrm{o} \% 20 \mathrm{da} \% 20$ qualidade $\& \mathrm{f}=$ false. Acesso em: 21 Jun. 2020.

OLIVEIRA, Otavio J. (org). Gestão da Qualidade - Tópicos Avançados. Cengage Learning Editores, 2003. Disponível em: https://books.google.com.br/books?hl=pt-

$\mathrm{BR} \& 1 \mathrm{r}=\& \mathrm{id}=\mathrm{S} 8 \mathrm{y} 8 \mathrm{DwAAQBAJ} \& \mathrm{oi}=\mathrm{fnd} \& \mathrm{pg}=\mathrm{PT} 18 \& \mathrm{dq}=\mathrm{gest} \% \mathrm{C} 3 \% \mathrm{~A} 3 \mathrm{o}+\mathrm{da}+$ qualidade $\&$ ots $=$ BRnVtcZ9uh\&sig=xJq7ckVUI3cJRJ6CVm41GM-

$\mathrm{hF} 5 \mathrm{Y} \# \mathrm{v}=$ onepage $\& \mathrm{q}=$ gest $\% \mathrm{C} 3 \% \mathrm{~A} 3 \mathrm{o} \% 20 \mathrm{da} \% 20$ qualidade $\& \mathrm{f}=$ false. Acesso em: $21 \mathrm{Jun}$. 2020.

PIMENTA, Laura Cunha Neto. APQP: Caracterização da Aplicação da Metodologia de Gestão de Desenvolvimento de Produtos em Fornecedores dos Setores de Linha Branca e Automotivo. 2009. Dissertação de Mestrado - Universidade Federal de São Carlos, São Carlos, 2009. Disponível em:

https://repositorio.ufscar.br/bitstream/handle/ufscar/3602/2549.pdf?sequence=1\&isAllowed= y. Acesso em: 22 Jun. 2020.

ROCHA, Juliana Rossi Pereira; SALERNO, Mario Sergio. O papel do APQP - Advanced Planning for Product Quality no desenvolvimento de produtos: Análise de casos na relação montadora-autopeças. Gest. Prod. São Carlos, v. 21, n. 2, p. 231-243, 2014. Disponível em: https://www.scielo.br/pdf/gp/v21n2/v21n2a02.pdf. Acesso em: 21 Jun. 2020.

VENTURA, Magda Maria. O Estudo de Caso como Modalidade de Pesquisa. Socerj, Rio de Janeiro. p 383-386, 2007. Disponível em:

https://d1wqtxts1xzle7.cloudfront.net/34829418/o_estudo_de_caso_como_modalidade_de_pe squisa.pdf?1411394806=\&response-content-

disposition=inline\%3B+filename\%3Dsetembro_outubro_O_Estudo_de_Caso_como_M.pdf\&

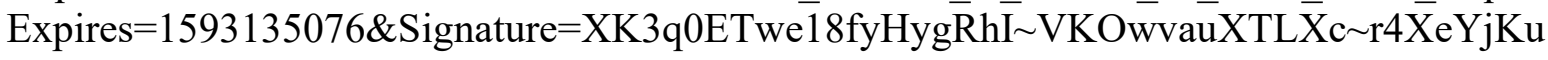
M5YqpGB1jiRg7B3fNDwkWw-8CApDBm kGsClaJ86pBXKaFISSS1 myG-

IFHATOQiIGeXI97zZH7QgSHok6E9-xPPHDKKex12BptlP0qCDJv9ntOJn48jCAenCKPE0066ZpoV470IlxezvPZbCQjyJZebpNJ5zopj2B 7yY3-

JfqqJUAL0tZv4aGO4rRMvTM0w RanPjWKuNCUBD4xW4pphQpo0q5fy5wJymHZ0U56a SzQg-tg9124A0wZerytpW610X60qnTRe9H-opbNMDEHXrs6DzKXLb9sSvxJrHFS5UQ_\&Key-Pair-Id=APKAJLOHF5GGSLRBV4ZA. Acesso em: 25 Jun. 2020. 